\title{
Visualization of aerosol particles generated by near infrared nano- and femtosecond laser ablation
}

\section{Working Paper}

Author(s):

Koch, Joachim; Schlamp, Stefan; Rösgen, Thomas; Fliegel, Daniel; Günther, Detlef

Publication date:

2000

Permanent link:

https://doi.org/10.3929/ethz-a-005707436

Rights / license:

In Copyright - Non-Commercial Use Permitted 


\section{Visualization of aerosol particles generated by near infrared nano- and femtosecond laser ablation}

J. Koch" \#, S. Schlamp ${ }^{+, \#, ~ T . ~ R o ̈ s g e n+, ~ D . ~ F l i e g e l ", ~ D . ~ G u ̈ n t h e r ~}{ }^{*}$

"Swiss Federal Institute of Technology, Laboratory of Inorganic Chemistry, Wolfgang-Pauli-Straße 10, ETH Zürich, CH-8093 Zürich, Switzerland

+Swiss Federal Institute of Technology, Institute of Fluid Dynamics, Sonneggstraße 3, ETH Zürich, $\mathrm{CH}-8092$ Zürich, Switzerland

\section{Abstract}

The expansion of aerosols generated by near infrared (NIR) nanosecond (ns) and femtosecond (fs) laser ablation (LA) of metals at atmospheric pressures was explored by laser-induced scattering. In order to achieve adequate temporal and spatial resolution a pulsed laser source was utilized for illuminating a $0.5 \mathrm{~mm}$-wide cross section of the expanding aerosol. It could, for instance, be shown that NIR-ns-LA under quiescent argon atmosphere provokes the formation of a dense aerosol confined within a radially propagating vortex ring. The expansion dynamics achieved under these conditions were found to be fairly slow whereas the degree of aerosol dispersion for NIR-ns-LA using helium drastically increased due to its lower viscosity. As a consequence, the maximum diameter of expansion differed by a factor of approximately four. The trajectories of aerosol particles generated by NIR-ns-LA using argon could, furthermore, be simulated on the basis of computational fluid dynamics (CFD). For this purpose, a model inspired by the thermal character of NIR-ns-LA taking into account a sudden temperature build-up of $10,000 \mathrm{~K}$ at the position of the laser focus was implemented.

In contrast, NIR-fs-LA generally resulted in extremely dynamic expansion patterns. Initial aerosol velocities derived from corresponding expansion plots varied from $10 \mathrm{~m} / \mathrm{s}$ up to $30 \mathrm{~m} / \mathrm{s}$ for fs-LA using argon and helium, respectively. Our results, moreover, indicate that fs-LA carried out under helium atmosphere favours a chaotic aerosol expansion. Analytical implications concerning, e.g. dispersion phenomena or the choice of the LA protocol and physical dimensions of future ablation cell designs are discussed.

Keywords: Femtosecond laser ablation; Aerosol visualization; Laser-induced scattering; Computational fluid dynamics; Chaotic expansion 
\#Corresponding authors:

J. Koch, E-mail koch@inorg.chem.ethz.ch, Tel.: ++41/44-633-6151; Fax: ++41/1-633-1071

S. Schlamp, E-mail:, schlamp@ifd.mavt.ethz.ch, Tel.: ++41/44-632-5124; Fax: ++41/44-632-1147 


\section{Introduction}

The utilization of femtosecond (fs) laser radiation has become a key technology for the progress of various applied and fundamental scientific disciplines. It has, e.g., been demonstrated that fs pulses enable to perform ultra precise, sub- $\mu$ m machining of conducting and non-conducting materials [1] or to examine the time-resolved reaction kinetics of molecular systems [2 - 4]. In recent years, there is furthermore a growing interest in the compositional analysis of aerosols generated by fs laser ablation (LA) of solid materials since corresponding particles were found to be "stoichiometric", i.e. exactly reflecting the composition of the sample to be analyzed [5]. Depending on the sensitivity required, the analysis of such aerosols is accomplished by either optical emission spectrometry (OES) or mass spectrometric (MS) detection using inductively coupled plasmas (ICP) [6 - 8].

In fact, the application of fs-LA as a source of "stoichiometric" aerosols has proven its capability in several studies $[5,9,10]$. Nevertheless, the utilization of nanosecond (ns)-LA still represents the most popular method of aerosol production due to its easy implementation and remaining difficulties of operating fs laser systems in the deep ultra-violet (UV) spectral range which is desirable for LA of highly transparent material. However, recent findings reveal ns-LA of metals and semi-conductors to provoke strong zone heating often resulting in material re-distribution and, thus, the release of "non-stoichiometric" aerosols [11].

To provide insight into mechanisms that control the formation of laser-produced aerosol particles, a series of supplementary studies dealing with plasma plume propagation and material ejection have been carried out [12 - 16]. Among these, Russo et al. [17] visualized the dynamics of plasmatic, i.e. non-particulate matter during the early stages of the ablation process using ns- and fs-LA and shadowgraphic imaging. Furthermore, Geohegan et al., Muramoto et al., and Nakata et al. [18 20] investigated the expansion of the particulate phase for delay times up to several milliseconds, i.e. far beyond the point where the condensational growth of particles is already finished. Within the scope of their studies, the authors were using laser-induced scattering for visualization allowing them to monitor even strongly dispersed aerosols. However, measurements were restricted to UVns-LA and low background pressures.

In contrast, less attention has been paid to the expansion of aerosols formed under atmospheric conditions even though information about the dynamics of particles interacting with higher background pressures would well complement the "patchwork"-like knowledge about LA and provide an adequate basis for (i) examining the consistency of models used to describe the aerosol formation and (ii) design optimization of conventional ablation cells which are being used for LA-ICP-MS/OES [21, 22].

This article reports on the visualization of near infrared (NIR) ns and fs laser-produced aerosols under atmospheric pressures on the basis of high-resolution, laser-induced scattering. Aerosols 
were monitored during the later stages of material ejection over a time-frame of 60 milliseconds under argon and helium atmospheres. In addition, aerosol expansion during the early stage of NIR-fs-LA was exemplarily examined for argon and delay times ranging from $10 \mu \mathrm{s}$ up to $5 \mathrm{~ms}$. Furthermore, the hydrodynamic expansion of aerosol generated by NIR-ns-LA was simulated by computational fluid dynamics (CFD) applying a model based on the thermal character of NIR-nsLA.

\section{Experimental}

\section{Aerosol generation}

A sketch of the experimental arrangement used for NIR-ns- / NIR-fs-LA and aerosol visualization is shown in Fig. 1. Both ns and fs pulses of approximately $1.5 \mathrm{~mJ}$ output energy and $10 \mathrm{~ns}$ and $150 \mathrm{fs}$ duration, respectively, were generated by the in [23] described Ti:Sapphire-based chirped pulse amplification (CPA) laser system emitting at a wavelength of $795 \mathrm{~nm}$ (Legend, Coherent Inc., Santa Clara, Ca, USA). Whenever ns pluses were used for LA the seed beam was simply blocked in front of the stretching unit. The laser radiation was delivered to a plano-convex lens ( $f=100$ $\mathrm{mm}$ ) using dielectric mirrors and subsequently focused onto the surface of a brass target positioned in the centre of a cylindrical, gas-filled ablation cell $\left(\mathrm{V}=50 \mathrm{~cm}^{3}\right)$. Throughout this study, measurements were generally performed under quiescent atmosphere.

\section{Aerosol visualization}

For aerosol visualization two quartz windows perpendicularly arranged to each other were mounted to the wall of the ablation cell. Their diameter was chosen to be $25 \mathrm{~mm}$ which enabled to access a clear aperture of around $20 \mathrm{~mm}$ times $20 \mathrm{~mm}$. Furthermore, a pulsed, frequency-doubled, and vertically polarized Nd:YAG laser (Minilite PIV, Continuum, Santa Clara, CA, USA) was employed as light source for the scattering set-up providing temporal resolution of $5 \mathrm{~ns}$. The utilization of vertically polarized laser radiation enabled to simultaneously access the Rayleigh ( $d<$ $0.05 \cdot \lambda)$, Debye $(0.05 \cdot \lambda<d<\lambda)$, and Mie $(d>0.05 \cdot \lambda)$ particle size range. Light scattered towards the observation facility, therefore, originated from particles whose size covers the entire spectrum generated by LA.

In order to adapt the beam diameter to the dimensions of the lateral cell windows, the radiation was feed through an optical assembly consisting of a cylindrical lens and a vertical slit. In this way, a narrow, $0.5 \mathrm{~mm}$ wide cross section could be illuminated. To avoid spurious contamination caused by aerosol particles from previous ablation events the cell was thoroughly flushed after each measurement. 
The scattered radiation was monitored through the observation window by a high-resolution (1024 x 1024 pixels), scientific grade CCD camera of 10 bit dynamic range (TM-1040, Jai Pulnix, San Jose, CA, USA) equipped with a macro objective. During image recording, unspecific radiation originating from the expanding plasma plume or residual reflections of the laboratory lightning was rejected by an interference filter $(\lambda=532 \mathrm{~nm}+/-5 \mathrm{~nm})$, positioned in between observation window and camera objective. Camera as well as $\mathrm{Nd}$ :YAG laser (flash lamp and Q-switch) were synchronized using a multi-channel delay box (LC880, Labsmith Inc., Livermore, CA, USA) which was triggered by the ablating laser pulse.

The laser specifications and experimental settings used for LA and aerosol visualization are given in Tab. 1. In the bottom part of Fig. 1, the timing scheme applied is shown. The maximum frame rate provided by the CCD camera used was limited to $17 \mathrm{~Hz}$, i.e. a continuous sequence of images could only be acquired with a time interval of $60 \mathrm{~ms}$. As a result, expansion studies for shorter temporal delays were generally assembled on the basis of independent measurements and do not belong to a single LA event. Nevertheless, the reproducibility of images acquired turned out to be sufficient to reconstruct the progression of aerosol trajectories and to perform velocimetric measurements within the time-frame considered.

\section{Results and discussion}

Regarding the literature published on the visualization of the LA process, most commonly shadowgraphic techniques have been utilized [e.g., 15 - 17]. Especially the implementation of laser-based set-ups offers a variety of advantages including high temporal resolution or the feasibility of monitoring any phenomenon that changes the refractive index such as shockwave propagation and density variations of expanding material in the initial stage of LA. However, the contrast achievable by shadowgraphy strongly decreases for less dense structures. It is, thus, inappropriate for observing dispersed nano-particles and agglomerates which represent the dominant part of aerosols formed. ${ }^{1}$ Moreover, depth resolution is missing since shadowgraphic images are line-of-sight measurements. Compared to this, laser-induced scattering provides depth resolution which is defined by the thickness of the light sheet passing through the aerosol.

NIR-ns-LA

In Fig. 2, the temporal progression of expanding aerosols generated by NIR-ns-LA using argon is shown. The focus of laser radiation was successively set to $2.0 \mathrm{~mm}$ above, at, and $2.0 \mathrm{~mm}$ below the target surface resulting in spot sizes of $100 \mu \mathrm{m}$ (focus at) and $400 \mu \mathrm{m}$ (defocused) and

\footnotetext{
${ }^{1}$ The contrast provided by laser-induced scattering even allows to visualize aerosols penetrating a strongly radiating plasma source unless the mean size of particles to be imaged falls below a critical value $(\sim \mu \mathrm{m})[24-26]$.
} 
fluences of $20 \mathrm{~J} / \mathrm{cm}^{2}$ (focus at) and $1 \mathrm{~J} / \mathrm{cm}^{2}$ (defocused), respectively. As can be seen, aerosols were captured in a radially moving vortex ring if the focus was kept above or at the surface. Surprisingly, no vertical material ejection could be observed. Furthermore, the particles remained strongly confined within a volume of approximately $0.01 \mathrm{~cm}^{3}$. Taking into account a moderate mass removal of $1-10 \mathrm{ng} / \mathrm{shot}$ and a mean particle size of $100 \mathrm{~nm}$, this value corresponds to a number density of $2 \cdot 10^{13}-2 \cdot 10^{14} \mathrm{~cm}^{-3}$ which, in turn, yields a particle collision frequency of $10^{4}-10^{5} \mathrm{~Hz}$ if a temperature of $300 \mathrm{~K}$ is assumed.

According to the literature, the application of NIR-ns-LA under argon atmosphere is known to generate a dense plasma right above the target due to strong absorption of the incident laser radiation (so-called plasma shielding). Depending on its viscosity such a plasma should be capable of preventing the aerosol from vertical expansion and, at the same time, acting as a source of lateral motion. More specific, the laser-induced plasma creates a spherical, outwards moving shock wave and flow, which overshadows the flow pattern produced by the expanding material. The aerosol particles ejected are thus pushed down and radially outwards, where they are captured in a vortex ring (see Fig. 2). In other words, the high temperature gradient in between laser-induced plasma and target surface creates a radially expanding gas stream right above the target surface which, eventually, results in a turbulence due to conservation of momentum.

To prove the consistency of this concept, the hydrodynamic expansion of a gas phase close to a hot spot was modelled by computational fluid dynamics (CFD). A sudden temperature build-up of $10,000 \mathrm{~K}$ was implemented simulating conditions typically achieved within an argon plasma that is generated in the focus of ns laser radiation. The resulting flow pattern is shown in the left panel of Fig. 3. Apparently, the gas phase is just about to roll up in a vortex after a time delay of $1 \mathrm{~ms}$, which is in good agreement with the temporal evolution presented in Fig. 2. To highlight the relatively slow moving flow around the vortex centre, a curved, black arrow was inserted into Fig 3. Of course, the applicability of the upper described model fails for irradiances insufficient to heat up the ambient gas by, e.g., reducing the pulse energy or avoiding an intermediate focus. Therefore, shifting the focus below the target surface stimulates the vertical aerosol ejection, as demonstrated by the third sequence of images shown in Fig. 2. The right panel of Fig. 3 illustrates the corresponding flow pattern, which was simulated without the implementation of a hot plasma but, otherwise, identical boundary and initial conditions. The aerosol now follows the expected, buoyancy-driven flow. For better representation of the simulated aerosol expansion, a movie clip showing animated velocity plots and flow patterns with and without assuming a hot spot is electronically supplemented to this article [Supp. 1].

Compared to this, NIR-ns-LA applying helium as expansion medium, shown in Fig. 4, always resulted in a vertical ejection of particles, independent on the relative focus position or fluence applied. Furthermore, the over-all dispersion turned out to be more dynamic. The maximum diameter of expansion for, e.g., NIR-ns-LA setting the focus below the surface was found to be four 
times as large compared to NIR-ns-LA using argon. In addition, no radially expanding aerosol fraction could be observed indicating the absence of a dense plasma above the surface.

NIR-fs-LA

Another sequence of images showing aerosol trajectories for NIR-fs-LA under argon atmosphere is presented in Fig. 5. Obviously, neither NIR-fs-LA above nor below the target surface gave rise to a vertical shielding as observed for NIR-ns-LA under equivalent conditions. However, tight focusing also provoked the formation of a vortex ring accompanied by a small, vertically moving particle jet. To determine the actual speed of expansion, the particle motion during the early stage of NIR-fsLA was exemplarily examined using argon for delay times ranging from $5 \mu$ s up to $5 \mathrm{~ms}$ (see Fig. 6). On the basis of the drag force model, which was found to yield best data correlation, an initial velocity of around $10-15 \mathrm{~m} / \mathrm{s}$ was derived. As can be seen in Fig. 7, the velocity, represented by the grey line, rapidly dropped in the course of expansion, falling below a value of $0.5 \mathrm{~m} / \mathrm{s}$ for delay times larger than $3 \mathrm{~ms}$. The corresponding stopping distance defined as the asymptotic value of vertical expansion amounted to about $9 \mathrm{~mm}$.

In Fig. 8, a sequence of images acquired for NIR-fs-LA using helium is depicted. As can be seen, NIR-fs-LA performed under these conditions always lead to extremely dynamic expansion patterns. However, the spatial distribution of aerosol particles appeared to be somewhat chaotic and consisted of multiple branches propagating into different directions. Assuming the expansion velocities for fs-LA using argon and helium to be proportional within the first $200 \mu \mathrm{s}$, the diameter of expansion shown in Fig. 6 suggests an initial speed which is about three times larger than that determined for argon.

\section{Analytical implications}

As already suggested above, the collision frequency of nano-sized particles produced by NIR-nsLA using argon as aerosol carrier remains fairly high after the LA process and amounts to more than $10^{4} \mathrm{~Hz}$. Most obviously, such aerosols are subject to pronounced coagulation and agglomeration. The results shown in Fig. 2 are, therefore, consistent with the increased production of larger, $\mu$-sized structures reported in [5]. Recent findings, moreover, indicate that dynamic expansion patterns as shown in Figs. 5 and 8, do not necessarily imply strong material deposition even if the dimensions of the cell used for extraction are smaller than the corresponding stopping distances. According to Garcia et al. [27], transport efficiencies of more than $80 \%$ can be achieved, independent on the individual cell volume or geometry chosen. Thus, the utilization of small cell volumes of, e.g., a few $\mathrm{mL}$ which often proves favourable for reducing the ICP-MS/OES signal dispersion does not generally result in poor transport efficiencies of aerosols released 
provided that there is no ejection of $\mu$-sized particles due to phase explosion and/or a direct contact of plasma plume and cell walls. These findings are in agreement with investigations previously carried out in [28] as well as own observations made in the scope of the present study. "Quick and dirty" experiments revealed that particles moving towards a flat surface appear to be rejected rather than being adsorbed. They, furthermore, tend to follow the streamlines of a complex flow pattern. Unfortunately, there is no information yet about the actual degree of material deposition. Nevertheless, a thorough examination of these phenomena is in preparation and will be published soon.

The over-all dispersion of laser-produced aerosols consists of (i) a kinetic part originating from the LA process itself and (ii) dispersion due to particle diffusion occurring during the transport period. The distance covered by diffusion can be calculated from the Einstein-Smoluchowski relation

$$
x(t)=\sqrt{\frac{k T \cdot t}{2 \pi \eta r}},
$$

where $\eta$ and $r$ denote the viscosity of the ambient gas and the particle radius, respectively. Taking into account the viscosity of helium at a pressure of $1 \mathrm{~atm}$ and particle radii ranging from 5 up to 50 $\mathrm{nm}$, the average diffusion speed after one second varies in between 25 and $80 \mu \mathrm{m} / \mathrm{s}$ for $\mathrm{T}=300 \mathrm{~K}$. Thus, diffusion-driven dispersion during the expansion can be disregarded compared to the kinetic part.

A stronger degree of dispersion can, therefore, only be achieved if a turbulent flow is established inside the ablation cell, passively, by a static inlet nozzle [28] or, actively, using a revolving device [29]. If no dispersive elements are implemented aerosols remain confined within cohesive fragments and filaments during their transportation to the ICP, similar to the plume of a candle burning off. A sequence of images illustrating such a collective motion over a time frame of about $0.5 \mathrm{~s}$ is shown in Fig. 9. Depending on the flow conditions right behind the ICP injector tube the entrainment of filamented aerosols may account for drastic density and temperature fluctuations of the ICP source during analysis which could give rise to ICP-induced elemental fractionation.

\section{Conclusion}

The expansion of aerosols generated by NIR-ns- and NIR-fs-LA of metals applying atmospheric pressures was explored by laser-induced scattering. It could be demonstrated that NIR-ns-LA performed under a quiescent argon atmosphere provokes the formation of a dense aerosol confined within a narrow, radially propagating vortex ring. Depending on the mass uptake assumed ( $1-10 \mathrm{ng} / \mathrm{shot}$ ), the local particle density varied in between $2 \cdot 10^{13}-2 \cdot 10^{14} \mathrm{~cm}^{-3}$. The trajectories of aerosol particles generated by NIR-ns-LA using argon could be simulated on the basis of CFD. To 
this end, a model inspired by the thermal character of NIR-ns-LA taking into account a temperature gradient between laser-induced plasma and target surface of 2,500 K was implemented.

The degree of aerosol dispersion achieved for NIR-ns-LA using helium atmosphere was found to significantly increase due to its smaller atomic mass, viscosity and, most probably, the higher thermal conductivity. As a result, the diameter of vertical expansion differed by more than one order of magnitude (a factor of four).

The utilization of NIR-fs-LA generally resulted in dynamic expansion patterns, independent on the kind of the surrounding gas. Initial aerosol velocities determined from expansion plots ranged from $10 \mathrm{~m} / \mathrm{s}$ up to $30 \mathrm{~m} / \mathrm{s}$ for fs-LA using argon and helium, respectively. Furthermore, the spatial distribution of aerosol particles released under helium turned out to be chaotic and consisted of multiple branches propagating into different directions.

According to the results documented in this paper NIR-ns-LA applying argon as aerosol carrier has to be regarded as the most unfavourable experimental configuration for suppressing the formation of larger particles since aerosols produced this way show a high spatial confinement and are, therefore, subject to pronounced coagulation and agglomeration. These findings are in agreement with the increased production of micrometer particles, recently found for NIR-ns-LA carried out using similar experimental conditions.

So far, less is known about the dispersion properties of laser-produced aerosols. However, the exploration of expansion patterns, trajectories, and initial velocities of aerosols originating from different LA protocols could help to prove the consistency of physical models that describe the ablation process beyond the stage of particle formation and, thus, to determine realistic input parameters for conventional or computer simulation-based design optimization of conventional ablation cells used for LA-ICP-MS/OES.

\section{Acknowledgements}

The comments made by two anonymous reviewers were very helpful to improve the quality of the manuscript and are gratefully acknowledged.

\section{References}

[1] B. N. Chichkov, C. Momma, S. Nolte, F. von Alvensleben, A. Tünnermann, Femtosecond, picosecond and nanosecond laser ablation of solids, Appl. Phys. A 63 (1996), 109 - 115

[2] M. J. Rosker, M. Dantus, A. H. Zewail, Femtosecond real-time probing of reactions: 1. The technique , J. Chem. Phys. 89 (1988), 6113 - 6127

[3] M. Dantus, J. Rosker, A. H. Zewail, Femtosecond real-time probing of reactions: 2. The dissociation reaction of ICN , J. Chem. Phys. 89 (1988), 6128 - 6140 
[4] R. B. Bernstein, A. H. Zewail, Femtosecond real-time probing of reactions: 3. Inversion to the potential from femtosecond transition-state spectroscopy experiments, J. Chem. Phys. 90 (1989), 829 - 842

[5] J. Koch, A. von Bohlen, R. Hergenröder, K. Niemax, Particle size distributions and compositions of aerosols produced by near-IR femto- and nanosecond laser ablation of brass , J. Anal. At. Spectrom. 19 (2004), 267 - 272

[6] A. L. Gray, Solid sample introduction by laser ablation for inductively coupled plasma source mass spectrometry, Analyst 110 (1985), 551 - 556

[7] D. Günther, B. Hattendorf, Solid sample analysis using laser ablation inductively coupled plasma mass spectrometry, Trends Anal. Chem. 24 (2005), 255 -265

[8] T. Pettke, C. A. Heinrich, A. Ciocan, D. Günther, Quadrupole mass spectrometry and optical emission spectroscopy: detection capabilities and representative sampling of short transient signals from laser-ablation, J. Anal. At. Spectrom. 15 (2000), 1149 - 1155

[9] J. Koch, H. Lindner, A. von Bohlen, R. Hergenröder, K. Niemax, Elemental fractionation of dielectric aerosols produced by near-infrared femtosecond laser ablation of silicate glasses, J. Anal. At. Spectrom. 20 (2005), 901 -906

[10] Q. Z. Bian, C. C. Garcia, J. Koch, K. Niemax, Non-matrix matched calibration of major and minor concentrations of $\mathrm{Zn}$ and $\mathrm{Cu}$ in brass, aluminium and silicate glass using NIR femtosecond laser ablation inductively coupled plasma mass spectrometry, J. Anal. At. Spectrom. 21 (2006), 187 - 191

[11] R. Hergenröder, O. Samek, V. Hommes, Femtosecond pulse shaping using a liquid-crystal display: Applications to depth profiling analysis, Mass Spectrom. Rev. 25 (2005), 086104

[12] W. Sdorra, K. Niemax, Temporal and spatial distribution of analyte atoms and ions in microplasmas produced by laser ablation of solid samples, Spectrochim. Acta B 45 (1990), $917-926$

[13] V. Margetic, T. Ban, F. Leis, K. Niemax, R. Hergenröder, Hydrodynamic expansion of a femtosecond laser produced plasma, Spectrochim. Acta B 58 (2003), 415 - 425

[14] D. B. Geohegan, Fast intensified-CCD photography of $\mathrm{YBa}_{2} \mathrm{Cu}_{3} \mathrm{O}_{7-\mathrm{x}}$ laser ablation in vacuum and ambient oxygen, Appl. Phys. Lett. 60 (1992), 2732 - 2734

[15] F. Kokai, K. Takahashi, K. Shimizu, S. lijima, Shadowgraphic and emission imaging spectroscopic studies of the laser ablation of graphite in an Ar gas atmosphere, Appl. Phys. A 69 (1999), 223 - 227

[16] J. H. Yoo, S. H. Jeong, X. L. Mao, R. Greif, R. E. Russo, Evidence for phase-explosion and generation of large particles during high power nanosecond laser ablation of silicon, Appl. Phys. Lett. 76 (2000), $783-785$ 
[17] X. Zeng, X. L. Mao, R. Greif, R. E. Russo, Experimental investigation of ablation efficiency and plasma expansion during femtosecond and nanosecond laser ablation of silicon, Appl. Phys. A 80 (2005), $237-241$

[18] D. B. Geohegan, A. A. Puretzky, G. Duscher, S. J. Pennycook, Time-resolved imaging of gas phase nanoparticle synthesis by laser ablation, Appl. Phys. Lett. 72 (1998), 2987 - 2989

[19] J. Muramoto, Y. Nakata, T. Okada, M. Maeda, Influences of preparation conditions on laserablated Si nano-particle formation processes observed by imaging laser spectroscopy, Appl. Surf. Sci. 129 (1988), 373 - 377

[20] Y. Nakata, J. Muramoto, T. Okada, M. Maeda, Particle dynamics during nanoparticle synthesis by laser ablation in a background gas, J. Appl. Phys. 91 (2002), 1640 - 1643

[21] J. Koch, G. Schaldach, H. Berndt, K. Niemax, Numerical simulation of aerosol transport, Anal. Chem. 76 (2004), 130A - 136A

[22] D. Bleiner, A. Bogaerts, Computer simulations of laser ablation sample introduction for plasma-source elemental microanalysis, J. Anal. At. Spectrom. 21 (2006), 1161 - 1174

[23] J. Koch, M. Wälle, J. Pisonero, D. Günther, Performance characteristics of ultra-violet femtosecond laser ablation inductively coupled plasma mass spectrometry at $~ 265$ and 200 nm, J. Anal. At. Spectrom. 21 (2006), 932 - 940

[24] K. Kahen, K. Jorabchi, C. Gray, A. Montaser, Spatial mapping of droplet velocity and size for direct and indirect nebulization in plasma spectrometry, Anal. Chem. 76 (2004), 7194 - 7201

[25] K. Jorabchi, K. Kahen, C. Gray, A. Montaser, In situ visualization and characterization of aerosol droplets in an inductively coupled plasma, Anal. Chem. 77 (2005), 1253 - 1260

[26] K. Jorabchi, R. G. Brennan, J. A. Levine, A. Montaser, Interferometric droplet imaging for in situ aerosol characterization in an inductively coupled plasma, J. Anal. At. Spectrom. 21 (2006), 839 - 846

[27] C. C. Garcia, H. Lindner, K. Niemax, Particle transport efficiency in femtosecond laser ablation inductively coupled plasma mass spectrometry applying $\mathrm{He}$ ablation cells with short and long washout times, accepted for publication (Spectrochim. Acta. B)

[28] D. Bleiner, D. Günther, Theoretical description and experimental observation of aerosol transport processes in laser ablation inductively coupled plasma mass spectrometry, J. Anal. At. Spectrom. 16 (2001), 449 - 456

[29] D. Bleiner, H. Altorfer, A novel gas inlet system for improved aerosol entrainment in laser ablation inductively coupled plasma mass spectrometry, J. Anal. At. Spectrom. 20 (2005), 754 - 756 


\section{Electronic supplementary}

Supp. 1 Temporal evolution of an expanding aerosol under argon atmosphere (i) close to a $10,000 \mathrm{~K}$ hot spot (right movie) and (ii) without the implementation of a hot spot (left movie) within a time frame of $1 \mathrm{~ms}$. The animation simultaneously shows the aerosol flow pattern and dynamics represented by normalized arrows. Grey shading of the arrows indicates the local gas velocity. Frames up to approximately $5 \mu$ s clearly show a pronounced shockwave propagation prior to the hydrodynamic motion 


\section{Tables}

Table 1 Laser specifications general experimental settings used for the visualization of NIR-nsand NIR-fs-LA-produced aerosols by laser-induced scattering

\section{Laser specifications}

Centre wavelength

$795 \mathrm{~nm}$

Pulse energy

$1.5 \mathrm{~mJ}$

Pulse duration

$150 \mathrm{fs}$ (seeded) / $10 \mathrm{~ns}$ (non-seeded)

Spot size

$400 \mu \mathrm{m}$ (defocused) / $100 \mu \mathrm{m}$ (focused)

\section{Laser-induced scattering}

Light source

Laser sheet thickness

Temporal resolution

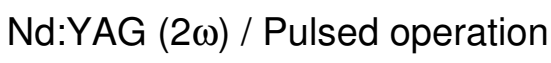

$0.5 \mathrm{~mm}$

$5 \mathrm{~ns}$ (equiv. to the laser pulse duration) 
Figures

Fig. 1 Sketch of the experimental arrangement used for aerosol visualization of aerosols produced by NIR-ns- / NIR-fs-LA. The ablation cell is not shown

Optical arrangement

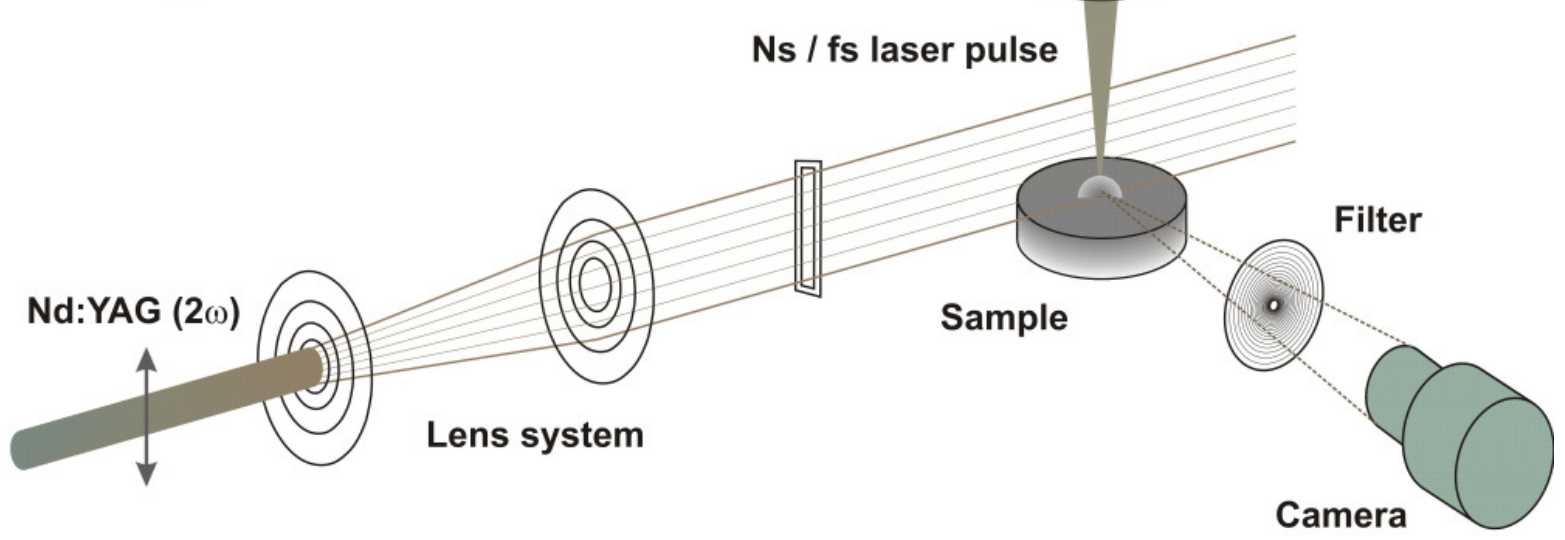

Trigger scheme

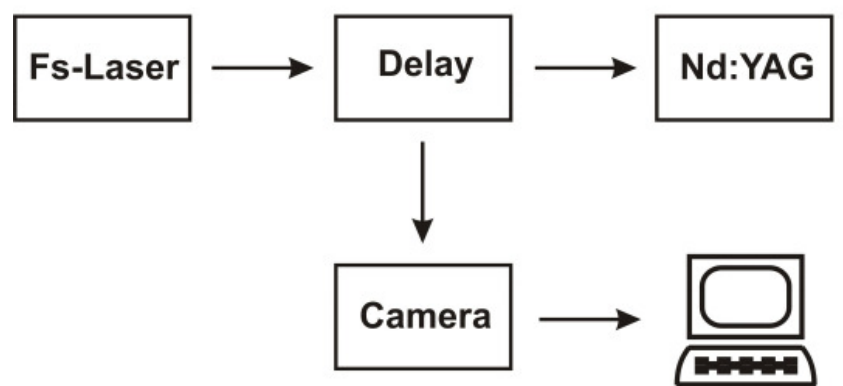


Fig. 2 Temporal progression of expanding aerosols generated by NIR-ns-LA using argon as aerosol carrier over a time frame of $63 \mathrm{~ms}$. The red sphere shown in the first sequence indicates the position of the laser focus. The second and third sequence refers to LA at and below the target surface, respectively

\section{(1) focus above}
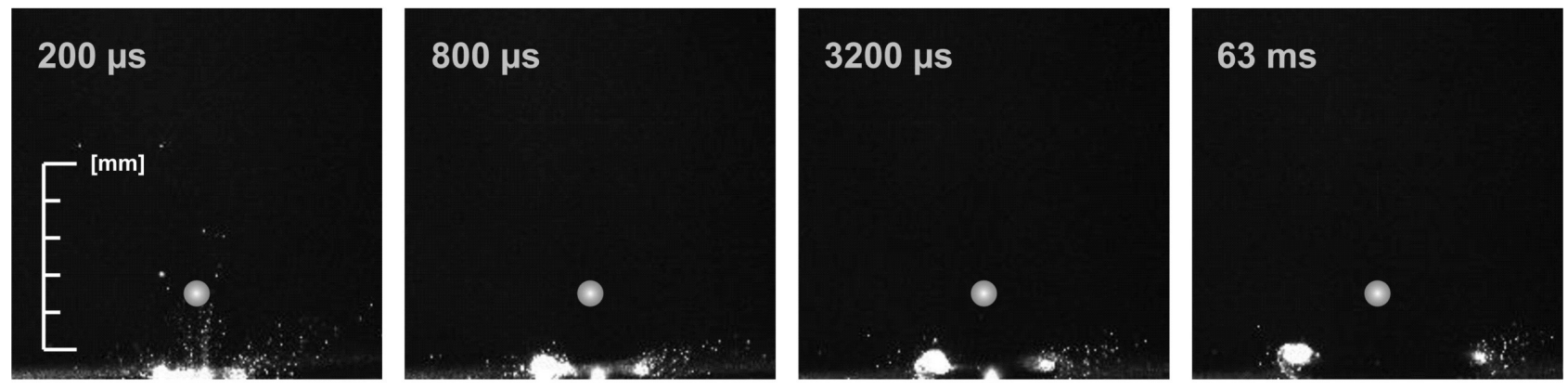

\section{(II) focus at}
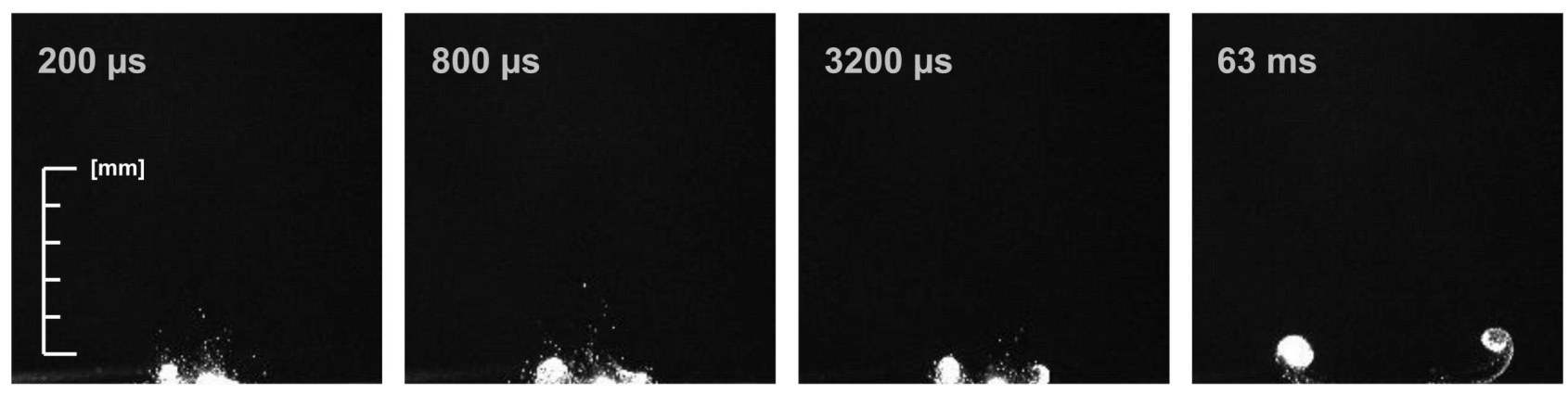

\section{(III) focus below}
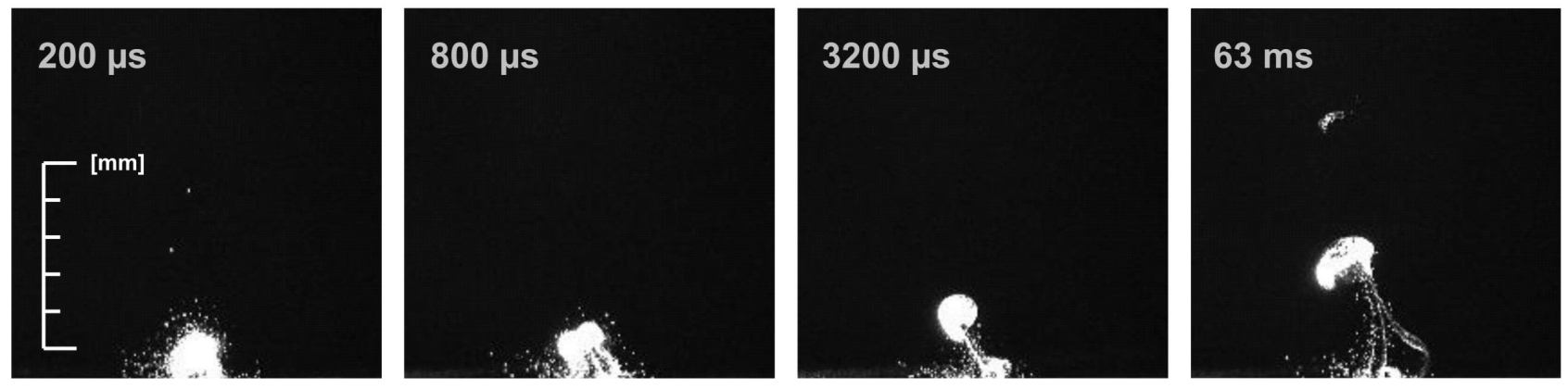
Fig. 3 Left panel (a): Hydrodynamic expansion of a gas phase close to an initially 10,000 K hot spot (diameter: $0.5 \mathrm{~mm}$, position: $2 \mathrm{~mm}$ above the surface) after $1 \mathrm{~ms}$ modelled by CFD. The surface (not shown) temperature was set to $2,500 \mathrm{~K}$ which equals the boiling temperature of $\mathrm{Cu}$. Red colouring corresponds to high aerosol concentrations, blue to low ones, i.e. ambient gas. Arrows represent the instantaneous flow field. The left edge is the axis of symmetry. Right panel (b): Hydrodynamic expansion without implementing a hot spot but, otherwise, identical boundary and initial conditions
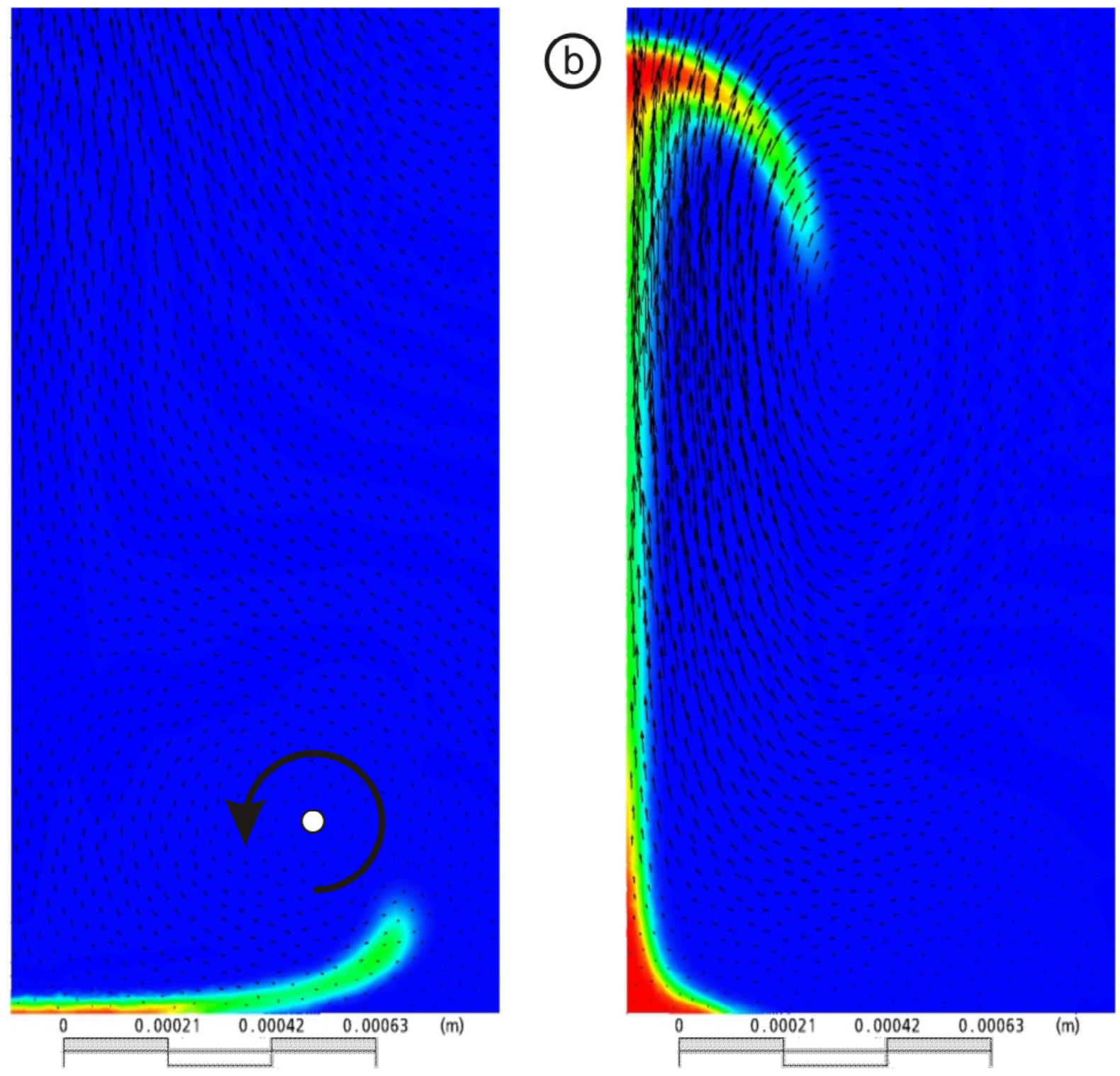
Fig. 4 Temporal progression of expanding aerosols generated by NIR-ns-LA using helium as aerosol carrier within a time interval of $63 \mathrm{~ms}$

(I) focus above
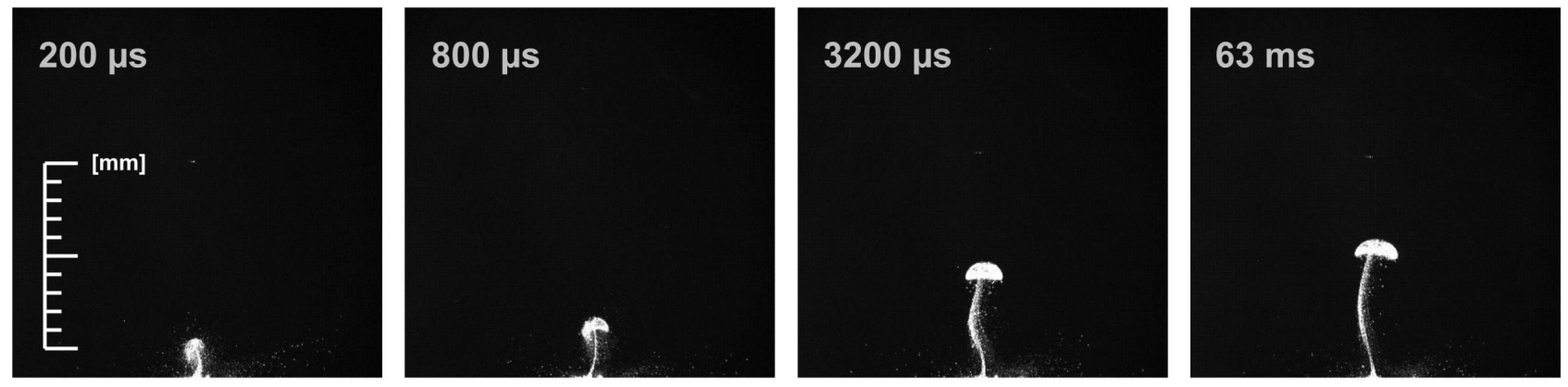

\section{(II) focus at}
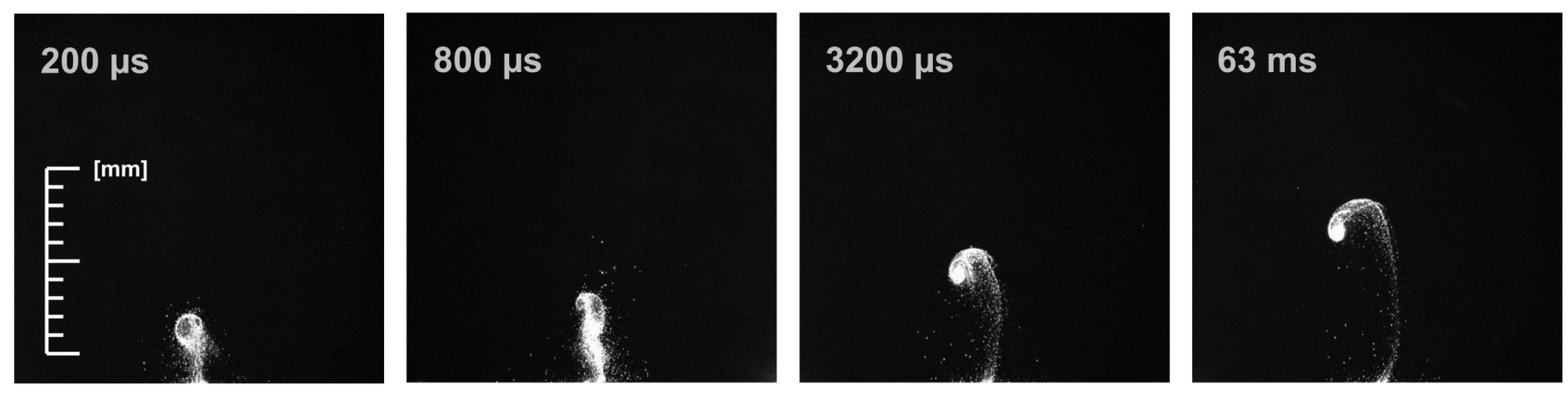

(III) focus below
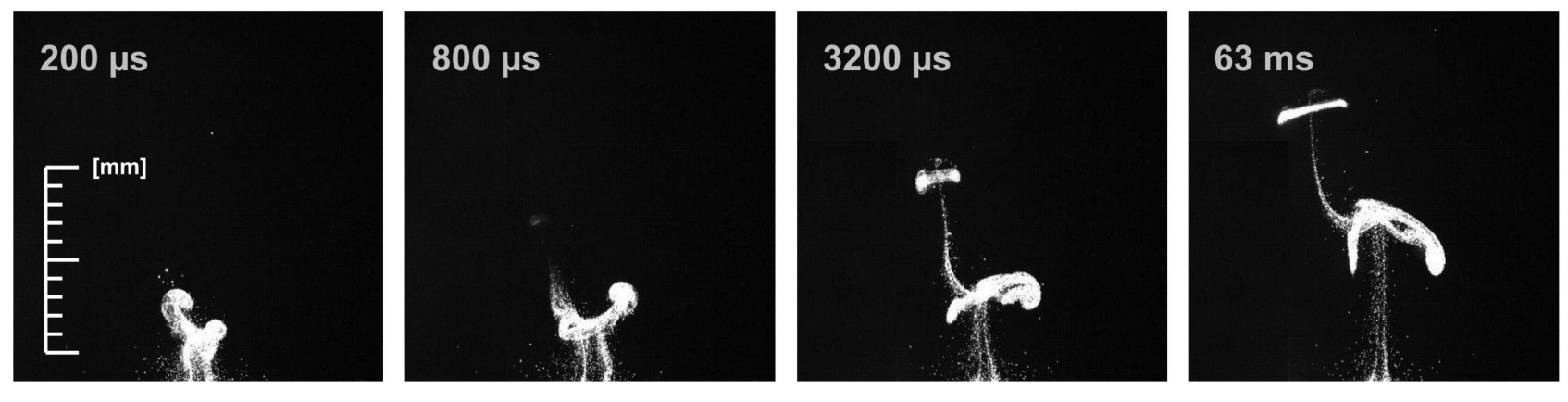
Fig. 5 Temporal progression of expanding aerosols generated by NIR-fs-LA using argon as aerosol carrier within a time interval of $63 \mathrm{~ms}$

(I) focus above
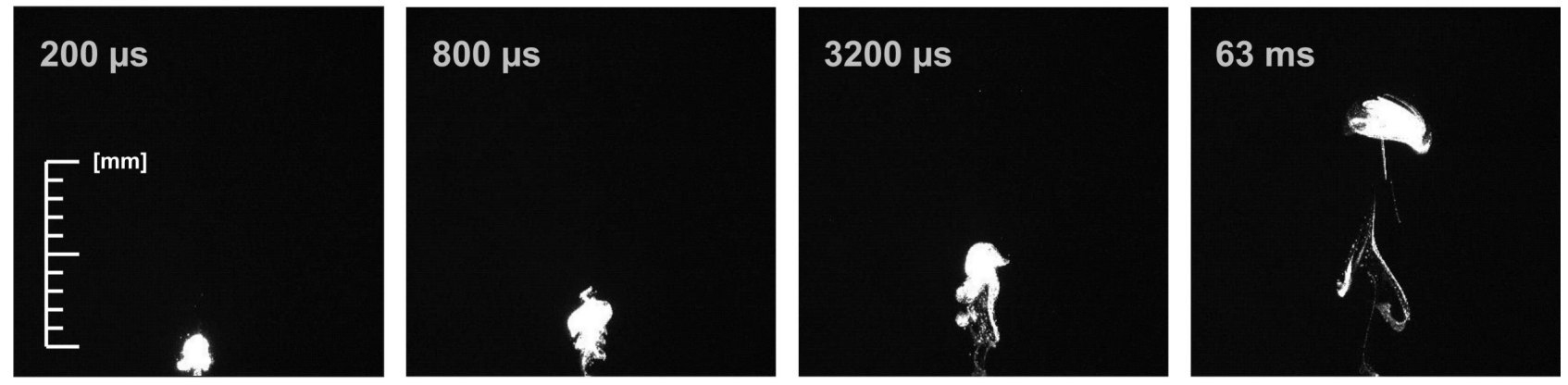

\section{(II) focus at}
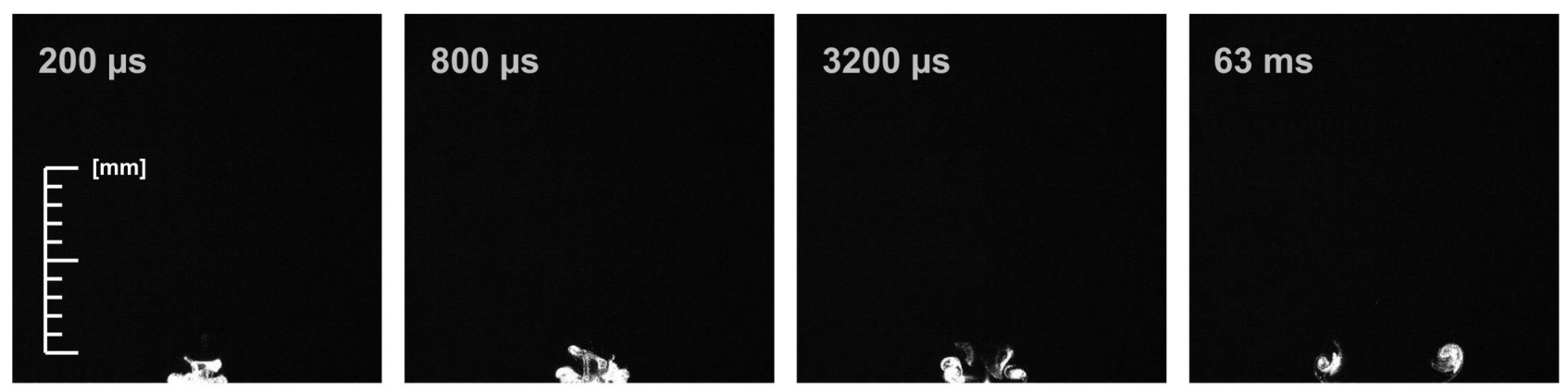

(III) focus below
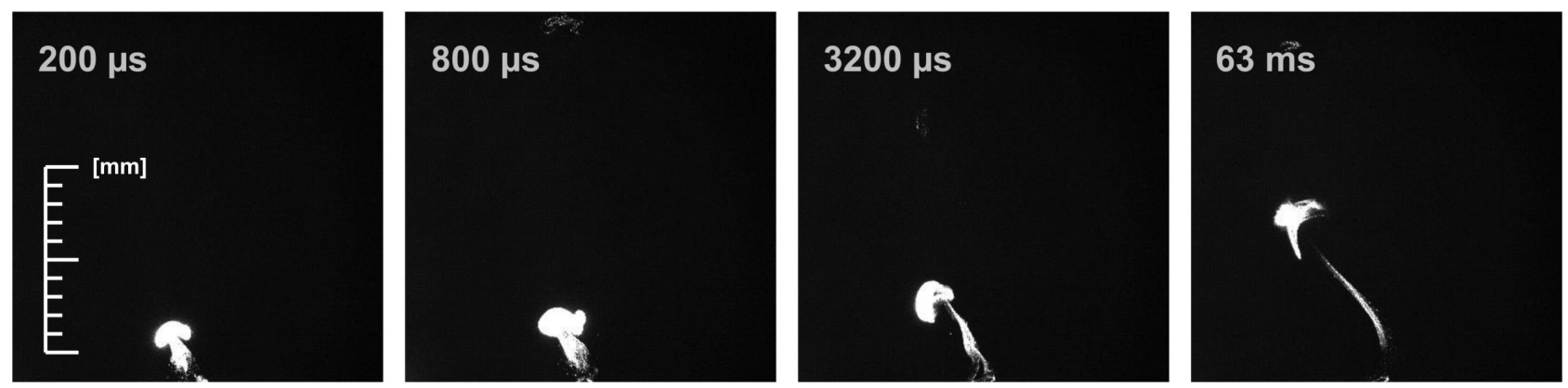
Fig. 6 Expansion study of an aerosol generated by NIR-fs-LA using argon for time delays up to $10 \mathrm{~ms}$. Please note, that the image acquired for $10 \mathrm{~ms}$ does not show the entire aerosol but only the tailing part. The red sphere shown in the first image indicates the position of the laser focus

NIR-fs-LA in argon / focus above
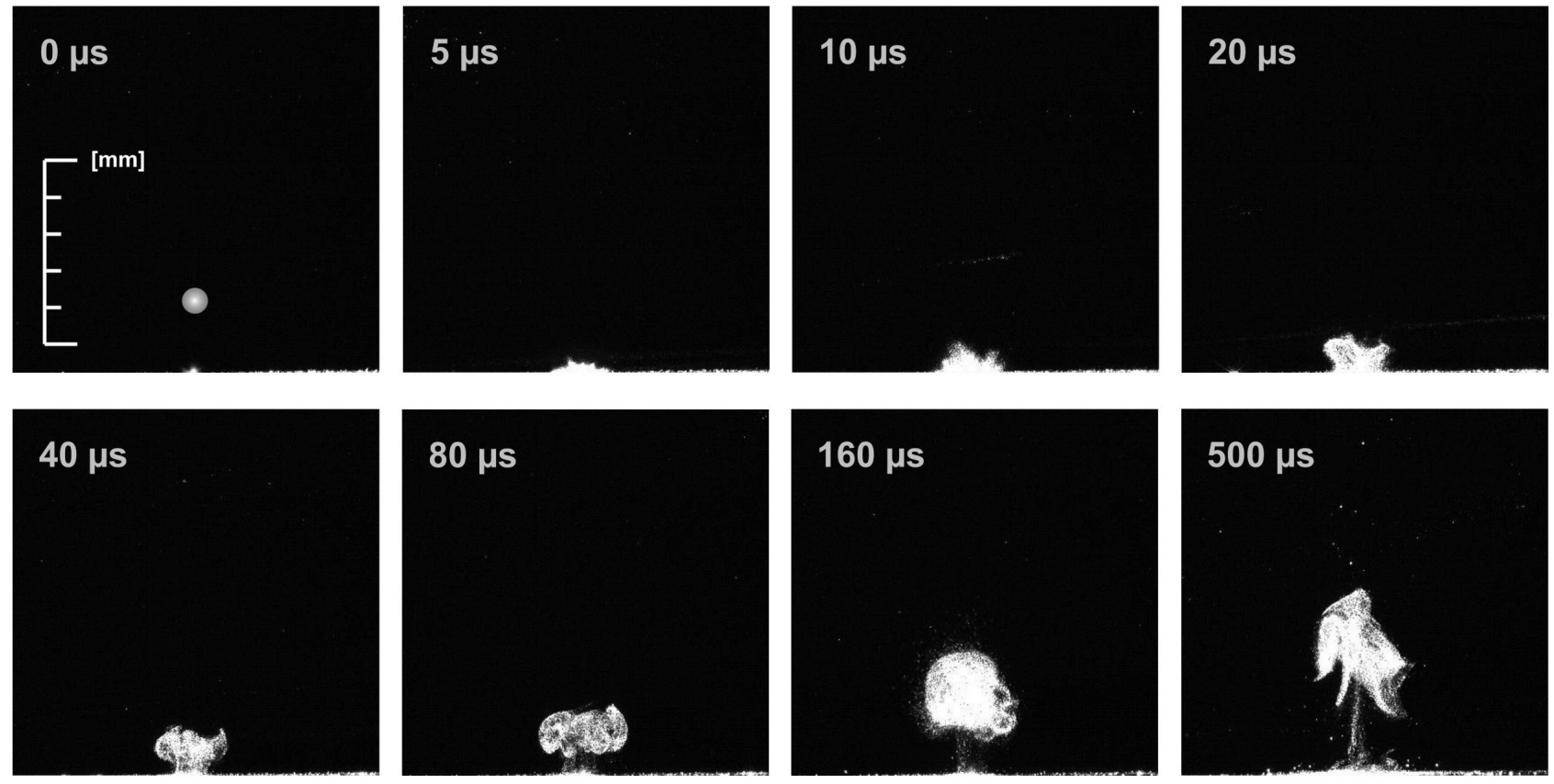

\section{$500 \mu s$}
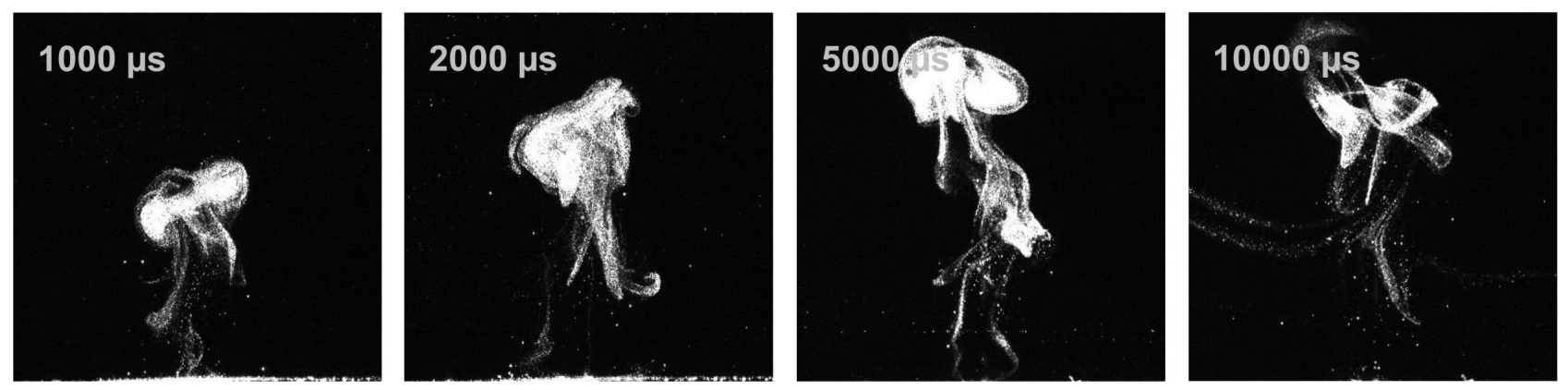
Fig. 7 Aerosol velocity derived from expansion plots for NIR-fs-LA under argon atmosphere acquired for delay times ranging from $10 \mu \mathrm{s}$ up to $5 \mathrm{~ms}$. Data points which were fitted to the drag force model function $s(t)=\frac{v_{0}}{\beta}\left[1-e^{-\beta t}\right]$ (grey line) refer to the maximum, i.e. vertical diameter of expansion. The corresponding velocity plot $v(t)$ (black line) was calculated from the $1^{\text {st }}$ derivative of $\mathrm{s}(\mathrm{t})$

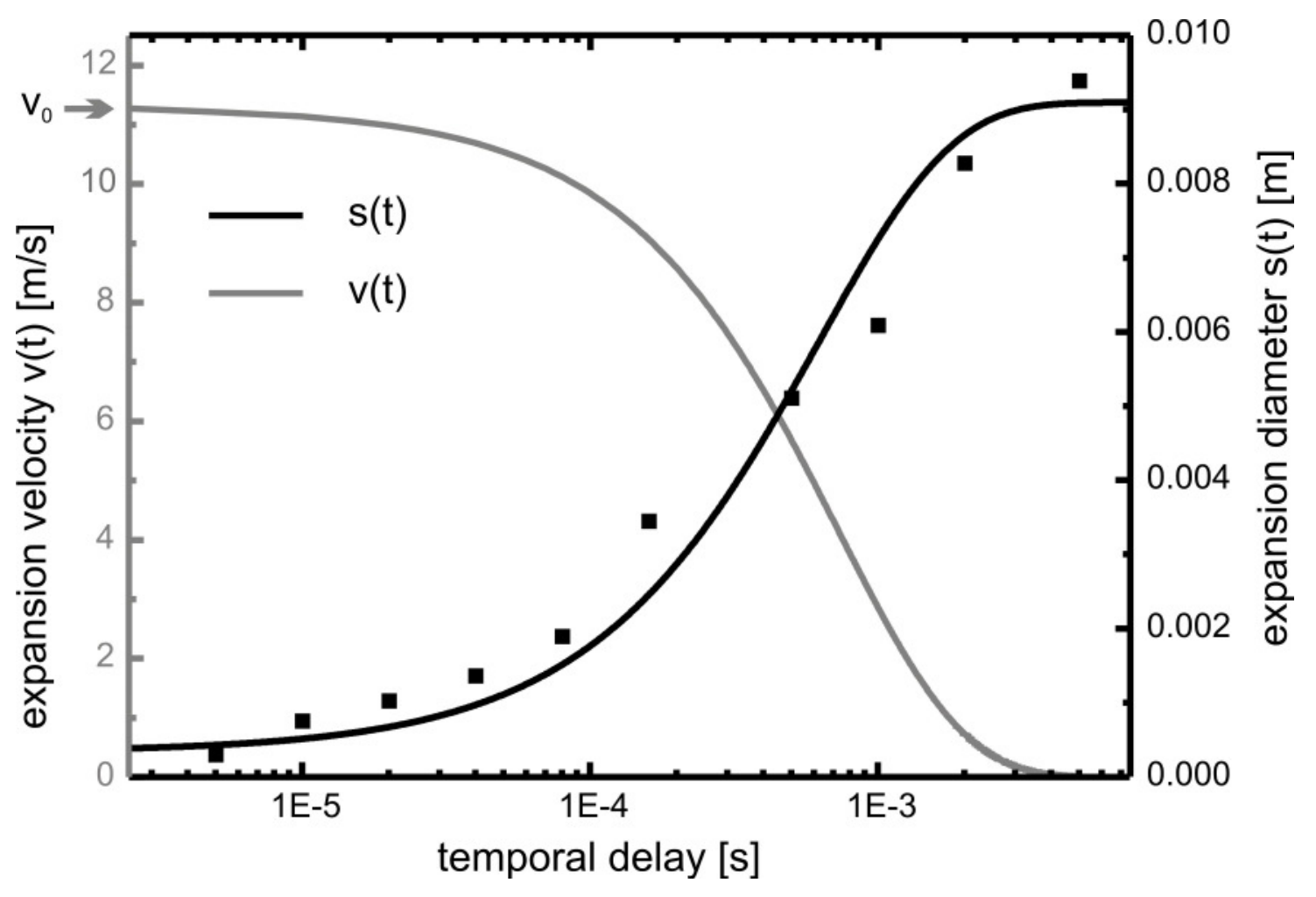


Fig. 8 Temporal progression of expanding aerosols generated by NIR-fs-LA using helium as aerosol carrier within a time interval of $63 \mathrm{~ms}$

(I) focus above
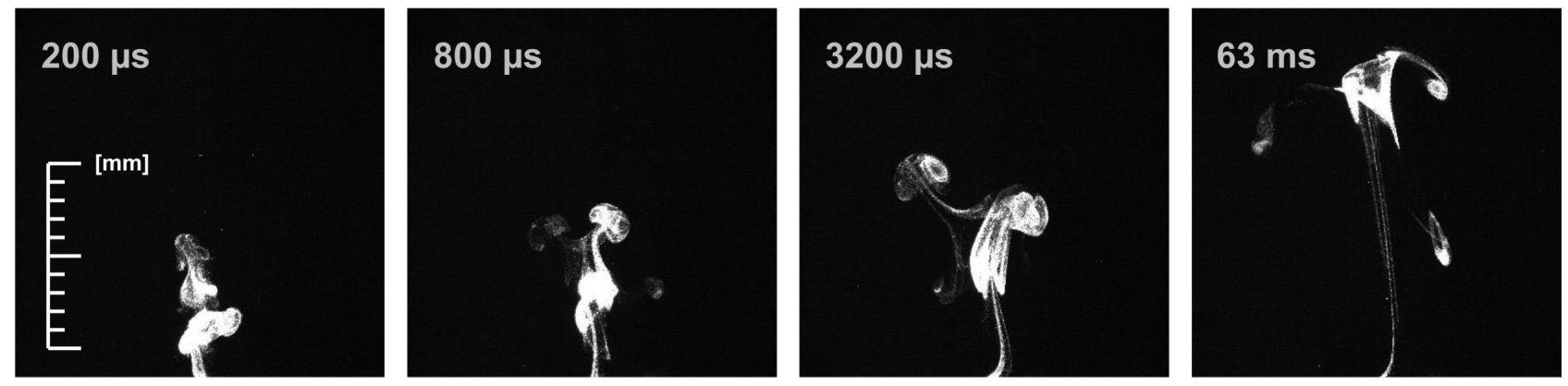

\section{(II) focus at}
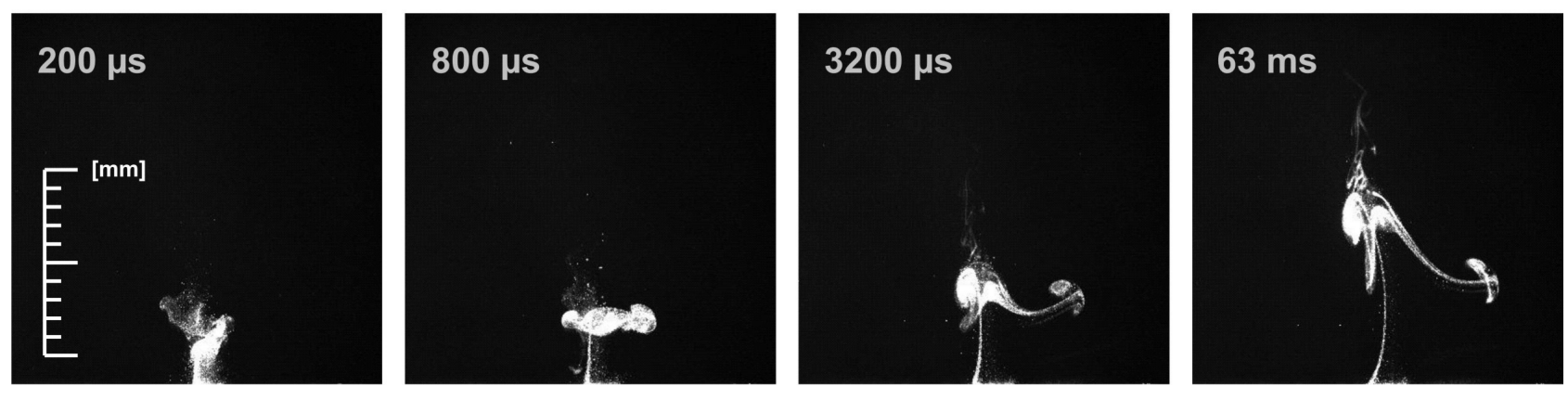

\section{(III) focus below}
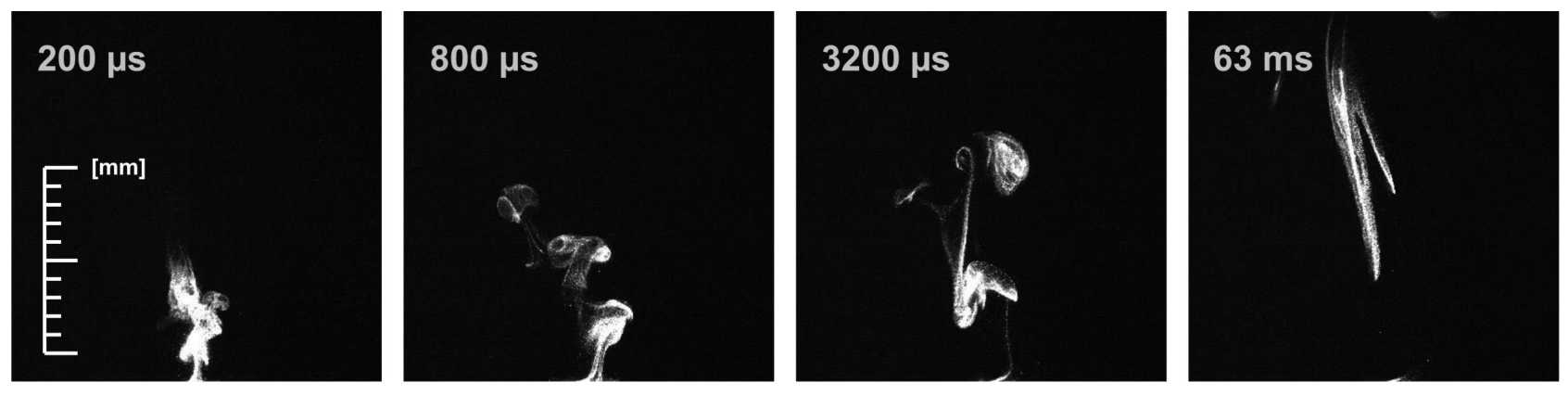
Fig. 9 "Long term" observation of aerosol particles generated by NIR-fs-LA of brass using atmospheric conditions (air) and a time period of $0.45 \mathrm{~s}$. The images clearly show a lateral movement of the filamented aerosol carried by an air flow of approx. $0.02 \mathrm{~m} / \mathrm{s}$. As expected, there is no dispersion of the filaments (see brownish arrows). The circles attached to the first frame $(\Delta \mathrm{t}=1 \mathrm{~ms})$ highlight large, $\mu$-sized particles ejected prior to the ultra-fine fraction or simultaneously but with a higher velocity. Their occurrence supports the existence of phase explosion which is being discussed as one possible mechanism of material removal during LA [16]

\section{NIR-fs-LA in air / focus above}
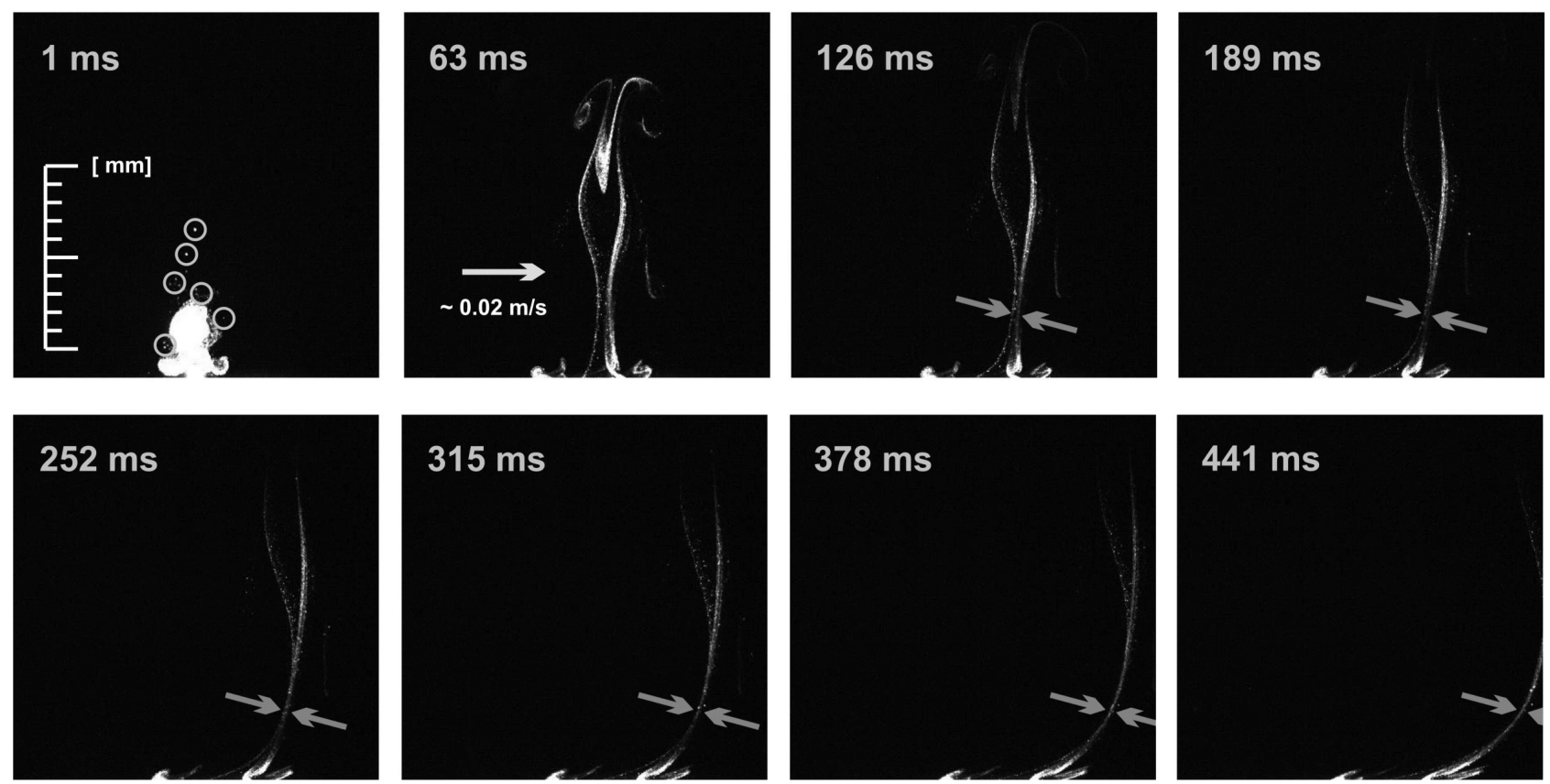

\section{$378 \mathrm{~ms}$}

\section{$441 \mathrm{~ms}$}

